\title{
Élaboration d'un modèle de prévision des risques d'infection du mirabellier (Prunus domestica L var insititia) par l'agent de la tavelure (Cladosporium carpophilum Thümen)
}

\author{
R Pineau, H Raymondaud, M Schiavon \\ ENSAIA-INPL, laboratoire de protection des cultures, 2, avenue de la Forêt de Haye, F-54500 Vandœuvre-lès-Nancy, France
}

(Reçu le 9 juillet 1990; accepté le 24 avril 1991)

\begin{abstract}
Résumé - Les conditions d'installation de la tavelure des arbres fruitiers à noyau (Cladosporium carpophilum Thümen) ont été étudiées sur mirabellier dans l'environnement climatique de Lorraine. La température optimale de germination des conidies du champignon in vitro est de $18^{\circ} \mathrm{C}$, mais le processus se déroule également correctement dans une gamme allant de $8^{\circ}$ a $22^{\circ} \mathrm{C}$. Les conidies en voie de germination supportent, entre 2 phases d'humectation, des périodes d'humidité relative basse (34\% HR) pouvant aller jusqu'à $10 \mathrm{~h}$.

Un modèle de prévision des infections du mirabellier par la tavelure est proposé : il est basé sur la durée d'humectation favorable à la germination du champignon et sur la température moyenne durant cette période critique. Ce modèle, testé avec succès durant 3 ans dans 11 vergers, peut servir de référence dans la lutte contre la maladie.
\end{abstract}

Cladosporium carpophilum / mirabellier / modèle de prévision / germination

Summary - Prediction of stone fruit scab infection periods on "Mirabelle" plum trees in the Lorraine. Infection conditions for stone fruit scab (Cladosporium carpophilum Thümen) have been studied in "Mirabelle" plum trees under climatic conditions in the Lorraine. Good germination of conidia occurs in vitro at $8-22{ }^{\circ} \mathrm{C}$ but is best at $18^{\circ} \mathrm{C}$. Germinating conidia are able to tolerate low relative humidity (34\% RH) exposures for $10 \mathrm{~h}$ between 2 moist periods. A predictive model for "Mirabelle" scab infection is proposed: it associates the length of the moistening period favorable for fungal growth and the average temperature during this critical period. This model has been tested successfully for 3 years in 11 "Mirabelle" plum tree orchards.

Cladosporium carpophilum / plum tree / predicting model / germination

\section{INTRODUCTION}

La tavelure des arbres fruitiers à noyau est une maladie redoutée par les producteurs de mirabelles de l'Est de la France. Cette variété de prune (Prunus domestica L var insititia) est en effet affectée par Cladosporium carpophilum dans des proportions pouvant aller jusqu'à $80 \%$ de fruits tachés à la récolte, en année très pluvieuse.

Le développement de cette maladie est encore mal connu : en 1976, dans une synthèse sur les maladies du mirabellier, Harranger ne fait état que des lésions sur rameaux et sur fruits. Viennot-Bourgin $(1949,1967)$ consi- comme bien plus importantes que celles observées sur fruits, alors que dans le cas du mirabellier les fruits jaune d'or sont particulièrement dépréciés par la présence des plaques noires provoquées par le champignon. Ceci constitue un réel facteur limitant à la commercialisation de la récolte comme fruit frais et pour la conserverie.

La maîtrise du problème passe obligatoirement par une connaissance approfondie de la biologie de l'agent pathogène responsable. C'est pourquoi les éléments de base nécessaires à l'établissement d'un modèle de prévision de l'infection ont été recherchés. 
Des travaux préliminaires nous ont montré que la forme du champignon qui sévit en Lorraine (forme imparfaite) a des exigences thermiques différentes des souches étudiées par Fisher (1961) en Australie ou par Lawrence et Zehr (1982) aux Etats-Unis. Ceci justifie une étude approfondie des conditions de germination des conidies émises au niveau des lésions sur les rameaux de l'année précédente qui constituent, sous le climat continental de Lorraine, la seule forme infectante de Cladosporium carpophilum (Schweizer, 1958; Raymondaud, 1988). Trois éléments ont été retenus parmi ceux susceptibles d'influer sur les premiers stades de développement du pathogène : durée d'humectation du substrat (feuilles ou fruits), température moyenne pendant la période d'humectation et variations d'humidité. Les effets de ces variables ont été étudiés dans des conditions proches de celles rencontrées sous climat continental.

Partant de ces données, une courbe de prévision des risques d'infection a été proposée et testée 3 années durant, en verger de production.

\section{MATÉRIEL ET MÉTHODES}

\section{Obtention des conidies}

Des rameaux de mirabellier de l'année, fortement tavelés, sont prélevés dans un verger âgé et non cultivé, situé à Lupcourt (54). Les prélèvements sont faits en début d'hiver, et le matériel est stocké à $4{ }^{\circ} \mathrm{C}$ jusqu'à la mise en incubation. Des fragments de rameaux porteurs de taches sont préalablement désinfectés à l'hypochlorite de sodium $1 \%$ durant $5 \mathrm{~min}$, puis rincés 2 fois à l'eau désionisée stérile. Ils sont ensuite placés en chambre humide (boîte de Petri dont le fond est rempli d'eau stérile) sur un support grillagé incurvé destiné à éviter le contact direct avec l'eau. Au terme de 8 j d'incubation à l'obscurité, à la température de $15{ }^{\circ} \mathrm{C}$, les conidies produites sont mises en suspension dans de l'eau distillée stérile; la concentration est amenée par dilution à $5.10^{3}$ conidies par $\mathrm{ml}$, valeur reconnue, à l'issue d'une expérience d'orientation, comme suffisante pour éviter d'éventuelles inhibitions.

\section{Principe du test de germination}

Les tests de germination sont effectués par dépôt d'un $\mathrm{ml}$ de la suspension de conidies sur un disque de filtration millipores ( $\varnothing$ des pores: $0,4 \mu \mathrm{m}$ ).

Les disques sont déposés sur un papier filtre (filtre Durieux sans cendre $n^{\circ} 111$ ) saturé d'eau au fond d'une boîte de Petri de $9 \mathrm{~cm}$ de diamètre.
À l'issue de différentes périodes d'incubation les conidies germées sont dénombrées sous microscope après coloration des filtres au bleu coton. L'état d'avancement de la germination est évalué par la longueur des tubes germinatifs classée selon les critères suivants :

- Classe 0, pas de germination ou début de gonflement, ou croissance limitée et anormale (amiboïde). Cette dernière forme correspond à un phénomène de dégénérescence rapide de la conidie;

- Classe $A$, longueur du tube germinatif inférieure à la moitié de la longueur de la conidie;

- Classe $B$, longueur du tube germinatif comprise entre la moitié et la longueur de la conidie;

- Classe $C$, longueur du tube germinatif comprise entre une fois et une fois et demie la longueur de la conidie;

- Classe $D$, longueur du tube germinatif supérieure à une fois et demie la longueur de la conidie.

Les dimensions des conidies mesurées à partir des populations obtenues sur rameaux tavelés sont les suivantes (longueur $x$ largeur/moyenne, en $\mu \mathrm{m}):(8,5-$ $15,5) \times(3,5-5,5) /(12-4,5)$. Les comptages sont effectués indifféremment sur les conidies mono- et biloculaires, qui peuvent également produire des tubes germinatifs. Par contre, les germinations de ramoconidies ne sont pas prises en compte.

Pour chaque test sont effectuées 4 répétitions comportant chacune un comptage de 100 conidies.

\section{Influence de la température}

Cinq températures sont testées dans une gamme définie à l'issue d'une expérience préliminaire : 8, 12, 15, 18 et $22{ }^{\circ} \mathrm{C}$ sous une photopériode journalière de $12 \mathrm{~h}$.

Le dénombrement des conidies germées est effectué toutes les 3 ou $4 \mathrm{~h}$ pendant $45 \mathrm{~h}$ et la première observation est réalisée au début de la sixième heure d'incubation.

\section{Influence de l'humidité relative}

Par analogie aux variations journalières auxquelles sont soumises les conidies au verger, 5 modalités d'expérience correspondant à des phases nocturnes saturées d'humidité entrecoupées d'une phase diurne plus sèche ont été testées sur disque millipore.

Les phases en humidité saturante sont réalisées suivant la méthode décrite préalablement; les périodes sèches sont obtenues en plaçant les disquettes support des conidies dans une enceinte réalisée suivant la technique de Young (1967) après un temps de séchage de 3 min sur un papier filtre sec. La solution saline choisie est le chlorure de magnésium donnant un taux de $34 \%$ d'humidité relative. Toutes les incubations sont effectuées à $18{ }^{\circ} \mathrm{C}$ sous une photopériode de $12 \mathrm{~h}$. 
Les modalités d'alternance retenues sont les suivantes:

- le lot témoin est soumis à une durée d'humectation continue de $22 \mathrm{~h}$;

- les autres lots (modalités 1 à 5) subissent 2 phases d'humectation de $11 \mathrm{~h}$ chacune séparées par une période d'humidité relative basse $(34 \% \mathrm{HR})$ de durée croissante. Cette phase dure $6 \mathrm{~h}$ pour la modalité $1 ; 8$ $\mathrm{h}$ pour la $2 ; 10 \mathrm{~h}$ pour la $3 ; 15 \mathrm{~h}$ pour la 4 et $20 \mathrm{~h}$ pour la 5. Les observations sont effectuées comme indiqué précédemment, à l'issue de la dernière phase d'humectation.

\section{Observation au verger}

Durant les années 1986, 1987 et 1988 des thermohygrographes «Richard" complétés par des «Kit" électroniques «STEFCE-INRA» (Pinguet, 1983) ont été installés dans 11 vergers naturellement contaminés et non traités durant le temps des observations.

Des relevés journaliers ont permis de mesurer la durée d'humectation et de calculer la température moyenne correspondant à cette période. Lorsque 2 périodes d'humectation sont séparées par une période sèche, cette phase est incluse dans la période critique si elle est inférieure ou égale à $2 \mathrm{~h}$.

On sait que, en Lorraine, la durée de la période de latence de la maladie sur fruit comme sur feuille, est de $18 \mathrm{j}$ à l'optimum thermique qui est de l'ordre de $19^{\circ} \mathrm{C}$. Cette durée peut s'élever à $30 \mathrm{j}$ et plus dans la gamme de températures moyennes observées, soit $12-22{ }^{\circ} \mathrm{C}$ (Rivano, 1982). D'après Bensaude et Keitt (1928) le champignon pénètre dans la plante environ 4 j après l'initiation de la germination. C'est pourquoi la vérification des périodes de contamination a été effectuée de la manière suivante :

De 8 à 10 j après le début d'une période supposée infectieuse, de jeunes fruits verts (100 à 200) sont prélevés dans des arbres voisins des appareils de mesure et placés dans une chambre stérile en atmosphère humide, à la température de $19^{\circ} \mathrm{C}$, sous une photopériode de $12 \mathrm{~h}$. Entre 15 et $25 \mathrm{j}$ après le début de la période critique, les fruits sont observés journellement et sont comptés comme contaminés ceux qui

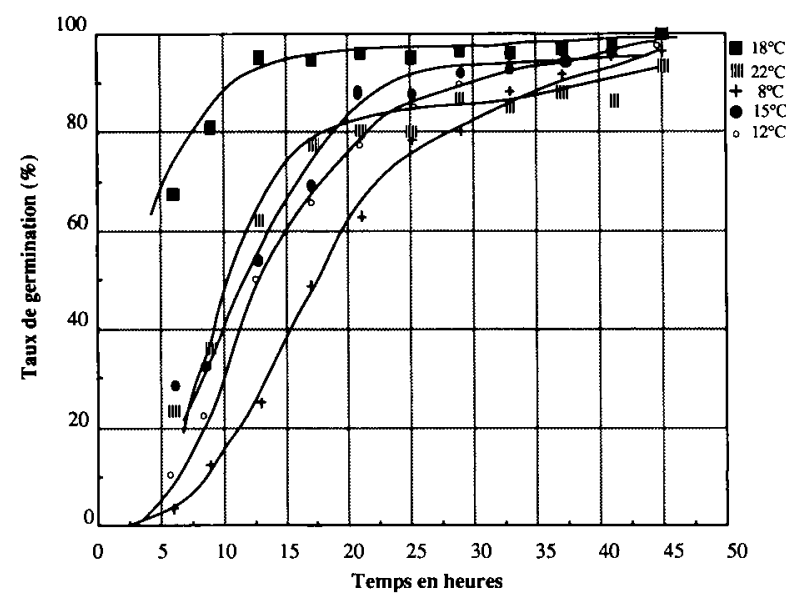

Fig 1. Taux de germination des conidies de Cladosporium carpophilum (souche lorraine) en fonction de la température. présentent les symptômes précurseurs de la maladie, soit par une identification visuelle (absence localisée de pruine qui est un symptôme précurseur de la maladie), soit après confirmation de la présence de conidiophores au microscope. Les observations sont poursuivies jusqu'à l'obtention des symptômes, ou sont arrêtées lorsque les fruits présentent un début de dégradation.

\section{RÉSULTATS ET DISCUSSION}

\section{Effet de la température}

\section{Analyse globale}

La figure 1 montre le taux global de germination des conidies de Cladosporium carpophilum, toutes classes confondues (A à D).

Dans toute la gamme des températures testées, ce taux est élevé puisque au terme de $45 \mathrm{~h}$ d'incubation, il varie entre $94 \%$ et $100 \%$.

C'est dans la dynamique de l'évolution du nombre de conidies germées que des différences marquées apparaissent. Si les courbes obtenues aux températures de 12,15 et $22{ }^{\circ} \mathrm{C}$ sont semblables, celle obtenue à $18^{\circ} \mathrm{C}$ est totalement différenciée et semble proche de l'optimum thermique requis pour la germination des conidies : plus de $60 \%$ des conidies ont germé dès la $6^{\mathbf{e}} \mathrm{h}$ d'incubation et $95 \%$ à la $13^{\mathrm{e}} \mathrm{h}$.

Ces résultats se démarquent nettement des données publiées antérieurement sur la germination de ce champignon. En effet, Bensaude et Keitt (1928) repris par Viennot-Bourgin (1947) font état d'un optimum thermique compris entre 20 et $27^{\circ} \mathrm{C}$. Lawrence et Zehr (1982) observent, au terme de $24 \mathrm{~h}$ d'incubation un meilleur taux de germination entre 25 et $30^{\circ} \mathrm{C}$. Par contre, ils n'observent aucune germination au-dessous de $10^{\circ} \mathrm{C}$ et moins de $20 \%$ à $15^{\circ} \mathrm{C}$, alors que dans des conditions analogues d'incubation (durée : $25 \mathrm{~h}$ ), nous dénombrons pour notre part respectivement $79 \%$ et $83 \%$ de conidies germées.

Lawrence et Zehr n'ont cependant compté comme germées que les conidies dont la longueur du tube germinatif est égale ou supérieure au diamètre de la conidie. Compte tenu des données concernant la mensuration des conidies du champignon (Ellis, 1971; Sivanesan, 1974; Raymondaud, 1988), ceci correspond aux 3 stades $B, C$ et $D$ définis précédemment.

Cette remarque nous a incité à examiner de façon plus détaillée le développement du tube germinatif. 


\section{Évolution qualitative de la germination}

La figure 2 donne l'évolution de la longueur des tubes germinatifs de $C$ carpophilum en fonction de la température et du temps d'incubation.

$\mathrm{Si}$ au terme de l'observation les pourcentages globaux des conidies germées sont comparables, la vigueur de la germination que l'on peut estimer par la longueur du tube germinatif est très variable : à $8{ }^{\circ} \mathrm{C}$, la majorité des conidies germées présente des tubes germinatifs au plus, égaux à la longueur de la conidie (stades $\mathrm{A}$ et $\mathrm{B}$ ) alors qu'à $15^{\circ} \mathrm{C}$ et $18^{\circ} \mathrm{C}$, ces 2 catégories sont minoritaires et l'on a essentiellement des tubes germinatifs d'une longueur supérieure à celle de la conidie.

Les travaux de Bensaude et Keitt (1928) indiquent que la pénétration du champignon à travers la cuticule intervient lorsque le tube germinatif atteint au moins la longueur de la conidie (classe $B$ et $C$ de nos travaux). Des observations (données non publiées) que nous avons effectuées sur des feuilles de mirabellier inoculées par des conidies de $C$ carpophilum, accompagnées de témoins sur disque de filtration, montrent que placées dans des conditions climatiques identiques, les conidies du champignon n'atteignent jamais le stade $D$ sur le vivant. Les stades $B$ et $C$ constituent donc l'élément déterminant pour estimer la possibilité de pénétration de $C$ carpophilum dans les tissus de la plante.

La figure 2 montre que le stade $B$ est atteint dès la $6^{\mathrm{e}} \mathrm{h}$ d'incubation à $18^{\circ} \mathrm{C}$; mais si pour les autres températures étudiées les phases initiales de la germination sont plus lentes, ce stade est atteint dès la $13^{\mathrm{e}} \mathrm{h}$ à 15 et $22^{\circ} \mathrm{C}$ et vers la $20^{\circ} \mathrm{à}$ $8^{\circ} \mathrm{C}$.

II est important de voir maintenant comment, durant ces laps de temps, les conidies en cours de germination vont supporter les variations hygrométriques constituées par des périodes d'humectation et de dessiccation relative, analogues à ce qui se produit au verger.

\section{Effet des alternances de périodes d'humecta- tion et de faible humidité relative}

La figure 3 présente les pourcentages de conidies germées à l'issue des différentes durées d'incubation. Toutes les combinaisons sauf les 2 modalités 4 et 5 qui présentent un nombre total de conidies germées très inférieur au témoin donnent un taux de germination égal ou supé- rieur à celui des conidies placées en permanence en condition d'humectation favorable.

Ainsi, il existe un seuil compris entre 10 et $15 \mathrm{~h}$ de dessiccation au-delà duquel la germination ne semble plus pouvoir reprendre : les conidies restent bloquées au stade où elles se trouvaient au terme de $11 \mathrm{~h}$ de développement et leurs tubes germinatifs dégénèrent (formes amiboïdes) et meurent.

En deçà de ce seuil et particulièrement pour une durée comprise entre 6 et $8 \mathrm{~h}$ de dessiccation, le processus de germination n'est pas stoppé puisqu'un plus grand nombre des conidies présentant un tube germinatif court (classe A) sont dénombrées par rapport au témoin. Ces traitements perturbent cependant la vitesse de développement du tube germinatif : la modalité $n^{\circ} 2$ présente 4 fois moins de conidies placées en classe $C$ et $D$ que le témoin.

Ces résultats peuvent être rapprochés de ceux de Schwabe (1980) qui a travaillé sur l'infection du pommier par des conidies de tavelure (Spilocaea pomi). Cet auteur a comparé l'effet de diverses modalités d'alternance de périodes d'humectation du feuillage séparées par des périodes d'hygrométrie basse comparables à celles que nous avons testées : de 32 à $53 \%$ d'humidité relative. II trouve que des intervalles de sécheresse pouvant aller jusqu'à $32 \mathrm{~h}$ consécutives entre les périodes d'humectation n'affectent pas la contamination par le champignon.

Dans ce cas, les durées cumulées des périodes d'humectation n'excèdent pas $8 \mathrm{~h}$.

Les exigences de Cladosporium carpophilum en la matière semblent être bien plus fortes puisque, en théorie, ce sont des durées d'humectation séparées par une période de moins de $10 \mathrm{~h}$ de forte dessiccation qui doivent être cumulées à celles-ci pour estimer le temps nécessaire à la germination des conidies.

Dans la pratique, de tels cas de figure sont rares mais il serait bon d'adapter au cas de la tavelure du mirabellier les travaux d'Olivier et al (1983) qui préconisent la prise en compte ou non, de la durée des périodes de nonhumectation en fonction de l'humidité relative de ces mêmes périodes.

\section{Prévision des périodes à risque d'infection}

À partir des données précédemment obtenues, nous avons établi les courbes suivantes (fig 4) donnant l'estimation des risques d'infection par 

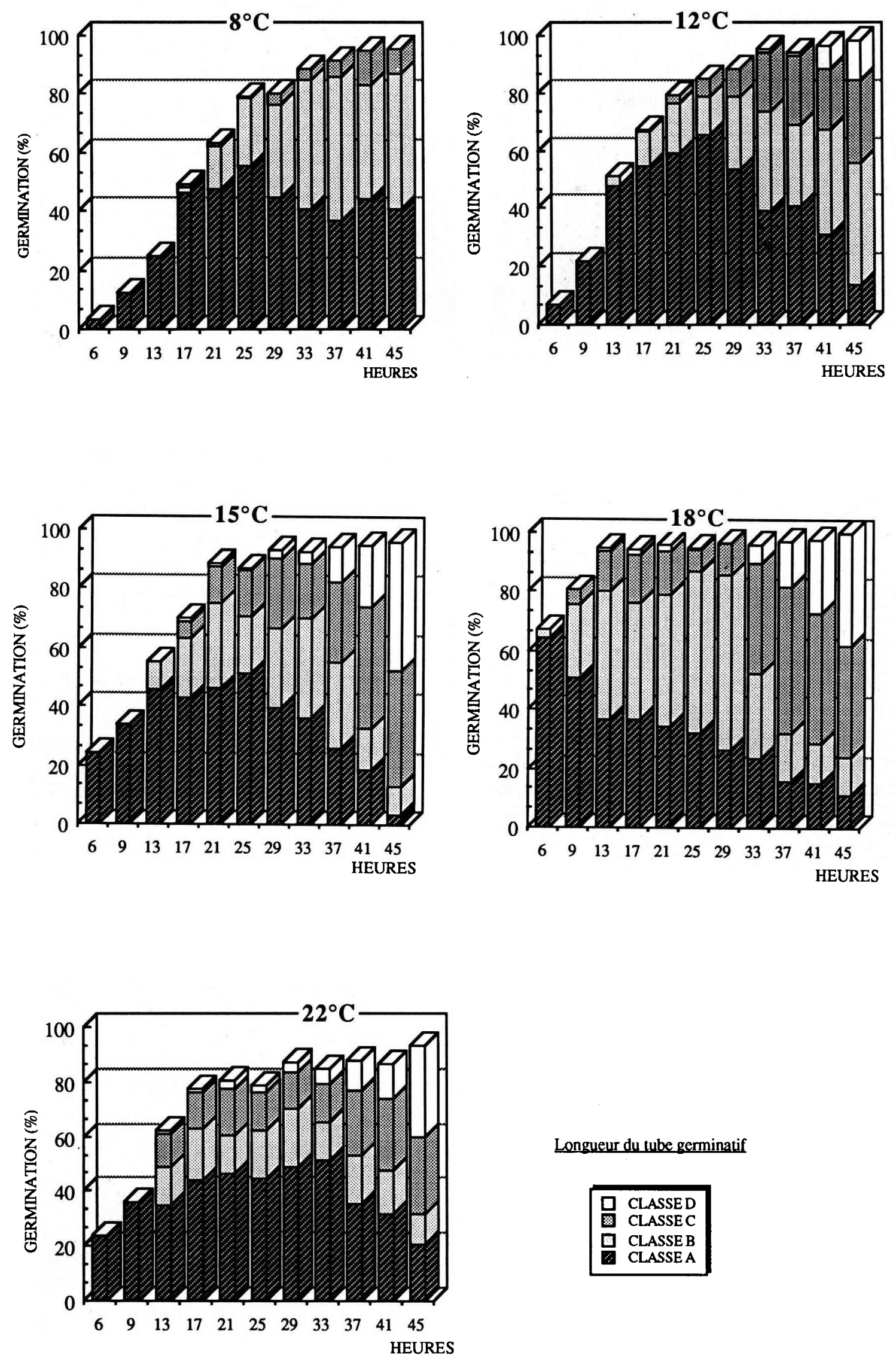

Longueur du tube germinatif

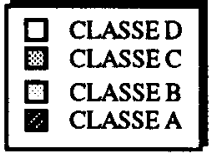

Fig 2. Effet de la température sur le développement des tubes germinatifs de Cladosporium carpophilum. 


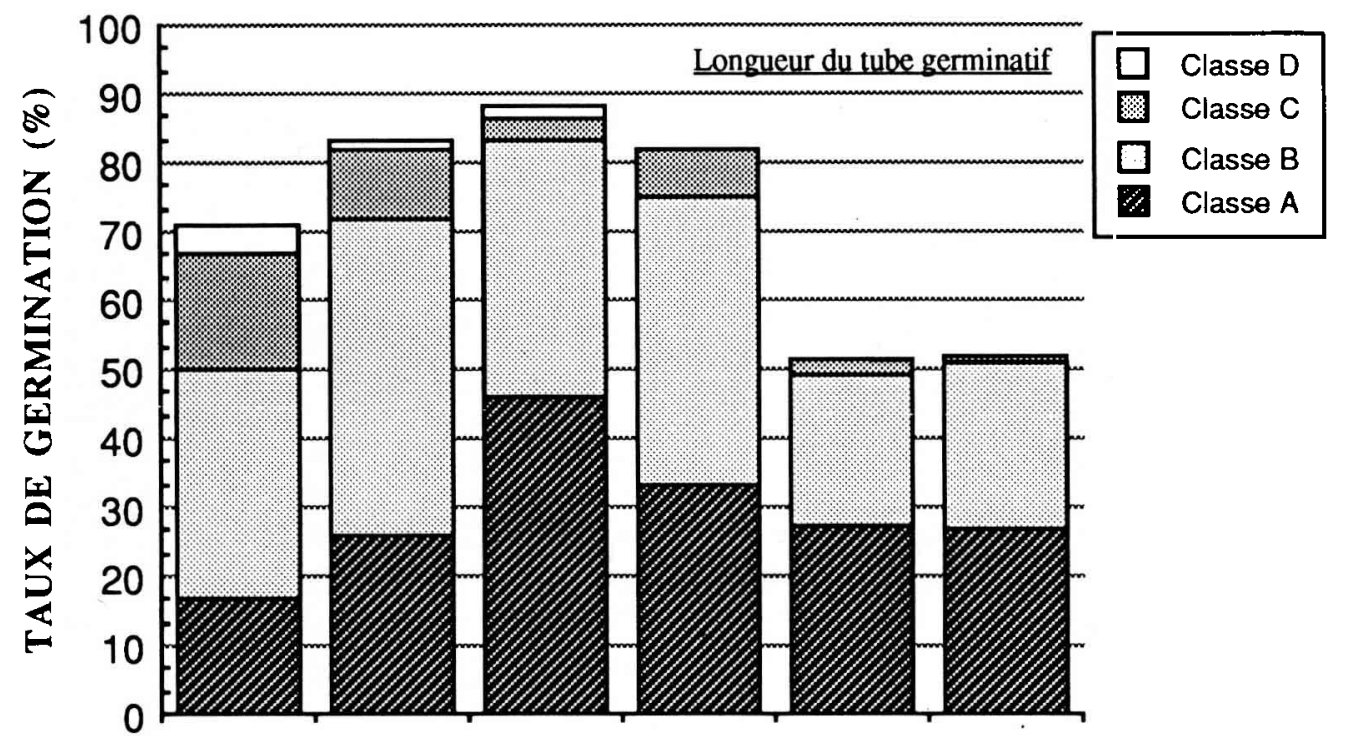

Humidité TEMOIN MOD.1 MOD.2 MOD.3 MOD.4 MOD.5 Modalités d'incubation

$\begin{array}{rrrrrrrr}100 \% & 22 & 11 & 11 & 11 & 11 & 11 & \text { 1 ère phase en heures } \\ 34 \% & 0 & 6 & 8 & 10 & 15 & 20 & \text { 2ème phase en heures } \\ 100 \% & 0 & 11 & 11 & 11 & 11 & 11 & \text { 3ème phase en heures }\end{array}$

Fig 3. Effet de l'alternance de périodes d'humectation et de dessiccation sur le développement des tubes germinatifs de Cladosporium carpophilum.

Cladosporium carpophilum en fonction de la durée d'humectation de la plante et de la température moyenne de cette période.

Ces courbes ont été construites à partir des taux de. germination du champignon au seuil de $25 \%$ (courbe basse) et $50 \%$ (courbe haute). En dessous de $25 \%$ de conidies germées, le risque d'infection est considéré comme faible, compte tenu du fait que nous n'avons observé à ce stade de développement du champignon que des tubes germinatifs de classe $A$.

Nous avons vu que selon les observations de Bensaude et Keitt (1928) la pénétration du champignon ne semble s'effectuer que lorsque la longueur du tube germinatif est au moins égale à la longueur de la conidie (à partir de la classe $B$ selon nos critères). Nous en déduisons que le risque d'infection est élevé lorsque ce stade est atteint; ce qui est toujours réalisé lorsque $50 \%$ de conidies sont germées.

La zone de risques modérés traduit l'insuffisance d'information que l'on a en matière de données comme la pression d'inoculum, la variabilité de la réceptivité de la plante, voire les limites d'utilisation des appareils de mesure.

Tout ceci a conduit à vérifier la validité du modèle ainsi proposé, ce qui a été réalisé durant 3 années consécutives en verger naturellement contaminé.

\section{Vérification de la validité du modèle}

Le tableau I donne la liste des stations dans lesquelles les observations ont été faites ainsi que le pourcentage de fruits présentant au moins une tache de tavelure en fin de saison.

Il convient ici de souligner la grande hétérogénéité du niveau d'expression de la maladie. Nous avons vérifié que dans tous les vergers existe un inoculum de $C$ carpophilum, par piégeage de conidies à l'aide de lames vaselinées. Mais, si dans tous ces vergers (sauf Watronville), nous avons pu constater l'existence de symptômes sur fruits, en proportion variable, au moins 1 année sur 3 , aucune tendance générale n'a pu être mise en évidence au niveau de la région.

Des disparités microclimatiques existent qui expliquent ponctuellement les infections et plaident, comme dans le cas des autres tavelures, en faveur d'un réseau densifié d'observation des données climatiques (Olivier, 1984).

La figure 5 donne une vue synthétique des relations établies a posteriori entre les observations en verger contaminé ayant conduit, ou non, à une infection des fruits et les données concernant la phase critique correspondante.

Sur un total de 86 périodes à risques, 33 ont conduit à l'apparition de symptômes sur fruits. 


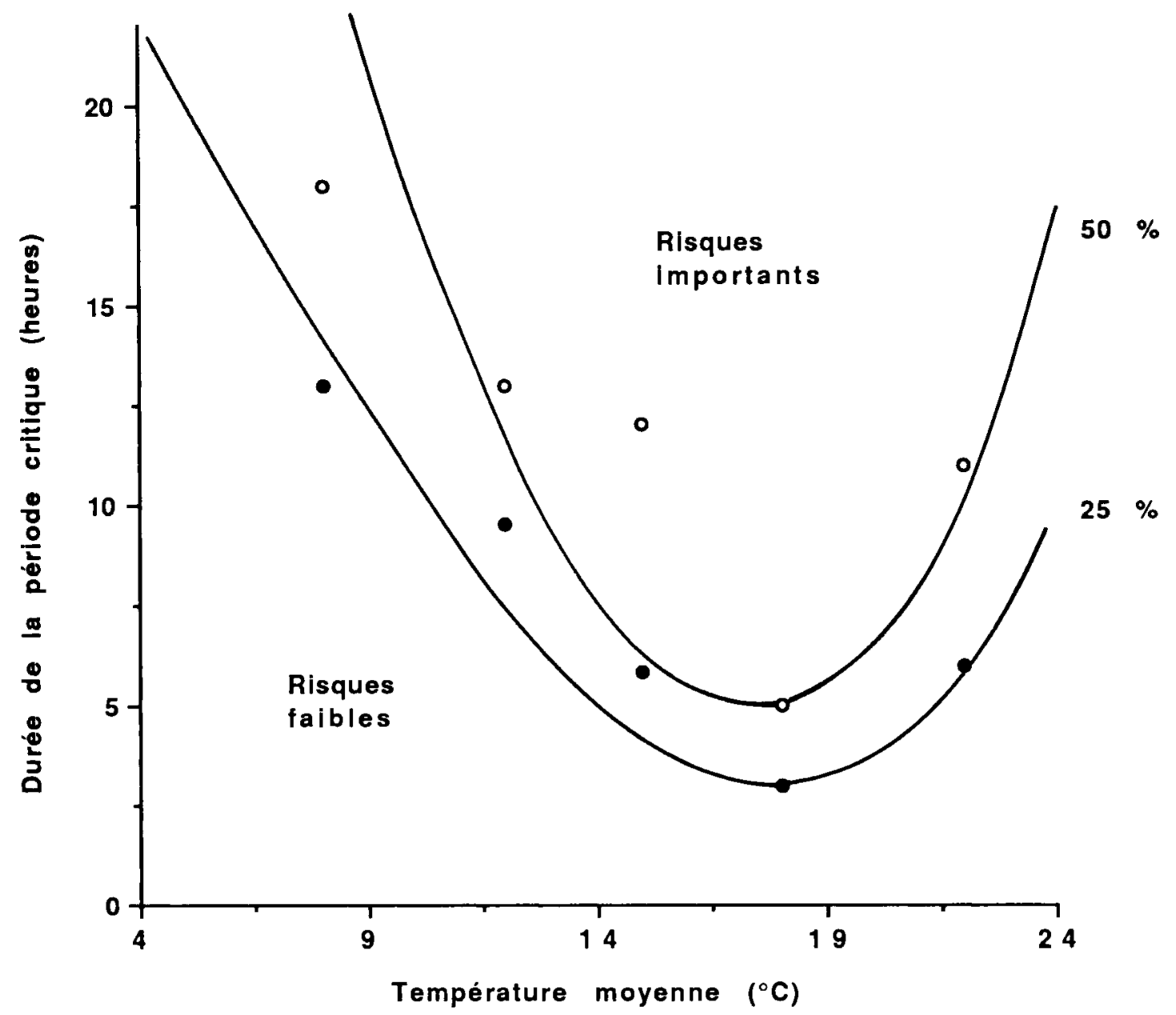

Fig 4. Estimation des risques d'infection du mirabellier par Cladosporium carpophilum basée sur les courbes ajustées aux seuils de $25 \%$ et $50 \%$ de taux de germination. - - O - courbe expérimentale ajustée, correspondant à $50 \%$ de conidies germées; - - courbe expérimentale ajustée, correspondant à $25 \%$ de conidies germées.

La figure 5 montre que toutes les observations ayant conduit à une contamination sont situées dans la partie du graphique prévoyant un risque d'infection élevé ou modéré. Aucune n'est située dans les zones à risque faible.
La contamination a pu s'effectuer même sous des températures basses, inférieures à $10^{\circ} \mathrm{C}$ mais avec, dans ces cas, des durées d'humectation voisines de (ou supérieures à) $20 \mathrm{~h}$. Nos essais de laboratoire ont montré que dans ces

Tableau I. Tavelure du mirabellier en Lorraine : localisation des vergers étudié et expression de la maladie.

\begin{tabular}{lllll}
$\begin{array}{l}\text { Situation du verger } \\
\text { Localité }\end{array}$ & Département & Année 1986 & $\begin{array}{c}\text { Pourcentage de fruits tavelés } \\
\text { Année 1987 }\end{array}$ & Année 1988 \\
\hline Lucey & 54 & 0 & 8 & 0 \\
Watronville & 55 & 0 & 0 & 0 \\
Vezon & 57 & 0 & 5 & 0 \\
Sion & 54 & 0 & 0 & 40 \\
Coyviller & 54 & 46 & non observé & 0 \\
Bulligny & 54 & $10-20$ & 20 & non observé \\
Buxieres & 55 & 10 & non observé & non observé \\
Chateau Salins & 57 & 0 & traces & non observé \\
Gugney & 88 & traces & non observé & non observé \\
Creue & 55 & non observé & traces & 0 \\
Rozelieures & 54 & non observé & 56 & 20
\end{tabular}


conditions, seules des conidies ayant atteint le stade B sont présentes (cf fig 2), ce qui confirme que le champignon est infectieux dès ce stade.

Aucune contamination n'a pu être mise en évidence quănd la température moyenne s'est élevée au-dessus de $20^{\circ} \mathrm{C}$, bien que, in vitro, le champignon atteigne les stades $B$ et $C$ dans des proportions notables $\left(>40 \%\right.$ ) dès la $13^{\mathrm{e}} \mathrm{h}$ d'incubation à $22^{\circ} \mathrm{C}$.

Ceci est expliqué par la raréfaction des périodes d'humectation prolongées en verger à partir de cette température.

Par ailleurs de nombreux points situés audessus de la courbe dite à risque fort n'ont produit aucun symptôme sur les fruits observés, bien qu'un inoculum potentiel ait été reconnu dans les vergers (taches sporulantes sur rameaux notamment). Ceci doit nous inciter à appronfondir la connaissance de l'état et du rôle de l'inoculum potentiel.

\section{DISCUSSION GÉNÉRALE}

S'il faut reconnaître la validité du modèle proposé, en tant qu'outil de prévision, il faut aussi tenter d'en améliorer la portée. Les seuls éléments de comparaison disponibles en la matière concernent la tavelure du pommier.

Les tables de Mills et Laplante (1951) ont constitué pendant longtemps les seules références de base pour la mise en œuvre de la lutte contre les tavelures des arbres fruitiers à pépins.

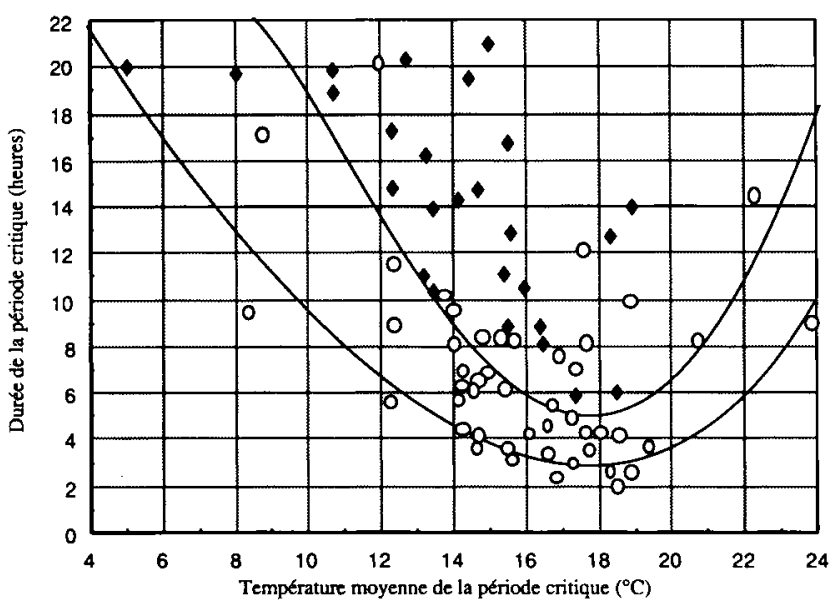

Fig 5. Vérification expérimentale a posteriori du modèle de prévision de la tavelure du mirabellier en verger de 1986 à 1988 : relevé des périodes d'humectation ayant ou n'ayant pas donné lieu à contamination. période ayant donné lieu à contamination; $O$ période n'ayant pas donné lieu à contamination; (courbe du modèle théorique de la figure 4).
Mais bien que permettant de prédire avec une bonne précision les périodes de contamination par le champignon, ces tables ont été critiquées (Sys et Soenen, 1970). C'est pourquoi leur emploi a été amélioré (Olivier et al, 1983; Olivier, 1984) notamment dans le but de répondre aux problèmes posés par les variations de réceptivité de la plante et l'état de l'inoculum, autant de facteurs que ne prennent pas en compte les tables de Mills.

Les données du présent travail sont le strict équivalent pour la tavelure du mirabellier des courbes de Mills pour celle du pommier. Mais la précision du modèle proposé mérite d'être affinée par de nombreuses autres expérimentations.

Dans cette optique plusieurs points doivent être envisagés :

- toutes les données relatives à la germination et à l'infection ont été obtenues soit in vitro, soit sur feuilles, organes relais de la maladie. Or, ni la réceptivité des rameaux de l'année sur lesquels se conserve le champignon, ni celle des fruits sous leurs différents états de maturité n'ont été étudiées à ce jour;

- à l'instar de ce qui est recommandé pour la tavelure du pommier (Olivier, 1984) il est probable que l'utilisation des courbes proposées doit être revue en fonction des situations à inoculum dense ou à inoculum faible;

- enfin la principale amélioration envisageable dans l'immédiat consiste en une meilleure prise en compte des périodes de non humectation entre 2 phases liquides.

Olivier et al (1983) préconisent pour la tavelure du pommier le cumul des phases humides et des périodes d'HR supérieures à $90 \%$. L'application de cet élément au cas de la tavelure du mirabellier permettrait de faire passer de nombreux points de nos graphiques de la zone à risques modérés à celle à risques forts.

De même, la prise en compte dégressive des périodes de moindre humidité devrait permettre aussi d'affiner la prévision.

Sur le plan pratique, le producteur de mirabelles a pendant longtemps lutté contre la maladie en ignorant l'état de l'inoculum et les risques réels d'infection : Harranger (1976) préconise 3 traitements à différents stades végétatifs de la plante (fin de la nouaison, fruit de $1 \mathrm{~cm}$, fruit formé mais vert).

Cette stratégie empirique a pu être améliorée et un système d'avertissements basé sur la connaissance des périodes à risque de contamination est en cours d'élaboration (Lemoine et al, 1990). 
Cependant, les modalités de pénétration dans la plante qui conditionnent le choix des produits fongicides restent encore inconnues pour $\mathrm{Cla}$ dosporium carpophilum, du moins pour les formes présentes en Lorraine dont la vitesse de croissance est très lente. C'est pourquoi des études sont en cours pour maîtriser l'inoculation de la tavelure du mirabellier et, ce faisant, améliorer encore le système de prévision des risques d'infection et l'efficacité de la lutte fongicide.

\section{CONCLUSION}

Aucune donnée n'existait jusqu'à présent pour cerner correctement les risques d'infection du mirabellier par la tavelure et utiliser de façon rationnelle les produits de traitement contre cette maladie.

La tavelure du mirabellier se démarque des tavelures des arbres fruitiers à pépins par le fait que son inoculum n'est constitué que par la forme conidienne, ce qui dans une certaine mesure, simplifie son étude.

II n'est pas possible non plus de la comparer aux tavelures des autres arbres fruitiers à noyau (pêcher, abricotier, amandier ni même prunier) observées par ailleurs sous d'autres climats : bien qu'il s'agisse du même agent pathogène, il semble que l'on ait affaire en Lorraine à un écotype adapté aux contraintes du climat continental.

La validité du présent travail se limite donc apparemment aux seules zones de culture étudiées, mais sur le plan méthodologique, nous sommes amenés à plusieurs réflexions de portée générale : tout d'abord, nous ne pouvons transposer directement au mirabellier les méthodes de lutte préconisées par ailleurs contre $C$ carpophilum sans de nouvelles études approfondies sur sa biologie.

C'est ce qui explique sans doute les échecs passés de la maîtrise du problème.

Le présent travail apporte des données de base à la connaissance des conditions d'installation du pathogène, mais nous nous heurtons encore à des aspects mal contrôlés (réceptivité de la plante, rôle du potentiel infectieux par exemple) largement étudiés sur d'autres cultures. D'autres aspects moins connus (relations avec la flore du phylloplan) pourraient venir non seulement aider à combattre Cladosporium carpophilum, mais d'une manière plus générale élucider certains comportements mal expliqués de pathogènes analogues.

\section{REMERCIEMENTS}

Nous tenons à remercier l'Association régionale d'expérimentation fruitière de l'Est (AREFE) et tout particulièrement Mme $C$ Gigleux et M H Ragon pour leur collaboration au niveau des observations et des expérimentations en verger.

\section{RÉFÉRENCES}

Bensaude M, Keitt GW (1928) Comparative studies of certain Cladosporium diseases of stone fruits. Phytopathology 18, 313-329

Ellis MB (1971) Dematiaceous hyphomycetes. CMI Kew, Surrey, $608 p$

Fisher EE (1961) Venturia carpophila sp nov, the ascigerous state of the apricot freckle fungus. Trans $\mathrm{Br}$ Mycol Soc 44, 337-342

Harranger J (1976) Les maladies du mirabellier. In: Le mirabellier (JJ Gerst, ed), INFLUVEC, Paris, 47-54

Lawrence EG, Zehr El (1982) Environmental effects on the development and dissemination of Cladosporium carpophilum on peach. Phytopathology 72, 773-776

Lemoine MP, Pineau R, Verbeke D (1990) Tavelure du mirabellier : vers la mise au point de la lutte raisonnée. Phytoma 417, 41-45

Mills WD, Laplante AA (1951) Control of diseases and insects in the orchard. NY Agric Exp Stn (Ithaca) Ext Bull 711, 21-27

Olivier JM (1984) Évolution de la lutte contre la tavelure du pommier. Déf Vég 225, 22-35

Olivier JM, Lambert C, Lefeuvre M (1983) Application du thermohumectographe KIT-INRA. Étude des risques de tavelure du pommier à l'échelle du Maineet-Loire (France). Bull OEPP 13, 47-56

Pinguet A (1983) Mesure de l'humectation : transformation d'un thermohygrographe et application à une station automatique agroclimatique. Bull OEPP $13,43-45$

Raymondaud $H$ (1988) La tavelure du mirabellier: étude biologique et épidémiologique de Cladosporium carpophilum Thümen et mise au point d'une méthode de lutte raisonnée. Thèse Inst Nat Polytech, Nancy, $161 \mathrm{p}$

Rivano F (1982) Mise au point d'une technique d'inoculation de Cladosporium carpophilum Thümen : application à l'étude épidémiologique de la tavelure du mirabellier. Mémoire DEA, INPL Nancy, $59 p$

Schwabe WFS (1980) Wetting and temperature requirements for apple leaf infection by Venturia inaequalis in South Africa. Phytophylactica 12, 69-80

Schweizer H (1958) Beiträge zur Biologie des Kirschen-und des Pfirsichschorferregers (Fusicladium cerasi (Rabh) Sacc Venturia cerasi Ad. und Cladosporium carpophilum v Thüm). Phytopathol $Z$ 33, 5598

Sivanesan A (1974) Venturia carpophila. CMI Descr pathogen Fungi Bact 402, Kew, Surrey, 2 p 
Sys S, Soenen A (1970) Investigations on the infection criteria of scab (Venturia inaequalis (Cooke) Wint) on apples with respect of the table of Mills and Laplante. Agricultura (Louvain) 18, 3-8

Viennot-Bourgin G (1947) Les tavelures des arbres fruitiers à noyau. Étude systématique et biologique. Fruits Outre-Mer 2, 170-178

Viennot-Bourgin G (1949) Megacladosporium carpophilum (Thümen). VB tavelure du pêcher, de l'amandier, de l'abricotier, du prunier. In: Les champignons parasites des plantes cultivées, T II, Masson, Paris, 1492-1502

Viennot-Bourgin G (1967) Les tavelures, In : Les champignons des arbres fruitiers à noyau. Ponsot, Paris, 39-55

Young JF (1967) Humidity control in the laboratory using salt solutions - a review. J Appl Chem 17, 241-244 